\title{
Gene profiling suggests a common evolution of bladder cancer subtypes
}

Donna E Hansel ${ }^{1 *}$, Zhongfa Zhang ${ }^{2}$, David Petillo ${ }^{2}$ and Bin T Teh ${ }^{3,4}$

\begin{abstract}
Background: Bladder cancer exists as several distinct subtypes, including urothelial carcinoma (UCa), squamous cell carcinoma (SCCa), adenocarcinoma and small cell carcinoma. These entities, despite showing distinct morphology and clinical behavior, arise from the urothelial lining and are often accompanied by similar precursor/in situ findings. The relationship between these subtypes has not been explored in detail.

Methods: We compared gene expression analysis of the two most common subtypes of bladder cancer, UCa $(n=$ 10) and SCCa $(n=9)$, with an additional comparison to normal urothelium from non-cancer patients $(n=8)$ using Affymetrix GeneChip Human genome arrays (Affymetrix, Santa Clara, CA). The results were stratified by supervised and unsupervised clustering analysis, as well as by overall fold change in gene expression.

Results: When compared to normal urothelium, UCa showed differential expression of 155 genes using a 5-fold cut-off. Examples of differentially regulated genes included topoisomerases, cancer-related transcription factors and cell cycle mediators. A second comparison of normal urothelium to SCCa showed differential expression of 503 genes, many of which were related to squamous-specific morphology (desmosomal complex, intermediate filaments present within squamous epithelium, squamous cornifying proteins, and molecules upregulated in squamous carcinomas from other anatomic sites). When compared, 137 genes were commonly dysregulated in both UCa and SCCa as compared to normal urothelium. All dysregulated genes in UCa were shared in common with SCCa, with the exception of only 18 genes. Supervised clustering analysis yielded correct classification of lesions in 26/27 (96\%) of cases and unsupervised clustering analysis yielded correct classification in 25/27 (92.6\%) of cases.

Conclusions: The results from this analysis suggest that bladder SCCa shares a significant number of gene expression changes with conventional UCa, but also demonstrates an additional set of alterations that is unique to this entity that defines the squamous phenotype. The similarity in deregulated gene products suggests that SCCa may be a much more closely related entity at the molecular level to conventional UCa than previously hypothesized.
\end{abstract}

Keywords: Gene expression profiling, Bladder cancer, Urothelial, Squamous, Subtype

\section{Background}

Urothelial carcinoma (UCa) represents the most common form of bladder cancer in the United States $(>90 \%)$ and is characterized by frequent mutations in TP53, $R B$ and PTEN [1]. Morphologically, UCa consists of invasive nests of carcinoma cells with variable atypia and frequent surrounding retraction artifact (Figure 1A), although this appearance can vary significantly. Less

\footnotetext{
* Correspondence: dhansel@ucsd.edu

'Department of Pathology, University of California at San Diego, 9500 Gilman Drive, MC 0612, La Jolla, CA 92093, USA

Full list of author information is available at the end of the article
}

common forms of bladder cancer in the United States include squamous cell carcinoma (SCCa; Figure 1B), adenocarcinoma and small cell carcinoma, which are defined as pure morphologic entities that lack a typical urothelial component [2]. Our understanding of the molecular relationships between these other forms of bladder cancer that arise from the urothelial lining has been limited and may be based on the low number of cases available for study and/or lack of significant attention paid to this topic. It appears, however, that despite a similar origin from the surface urothelium, these various 

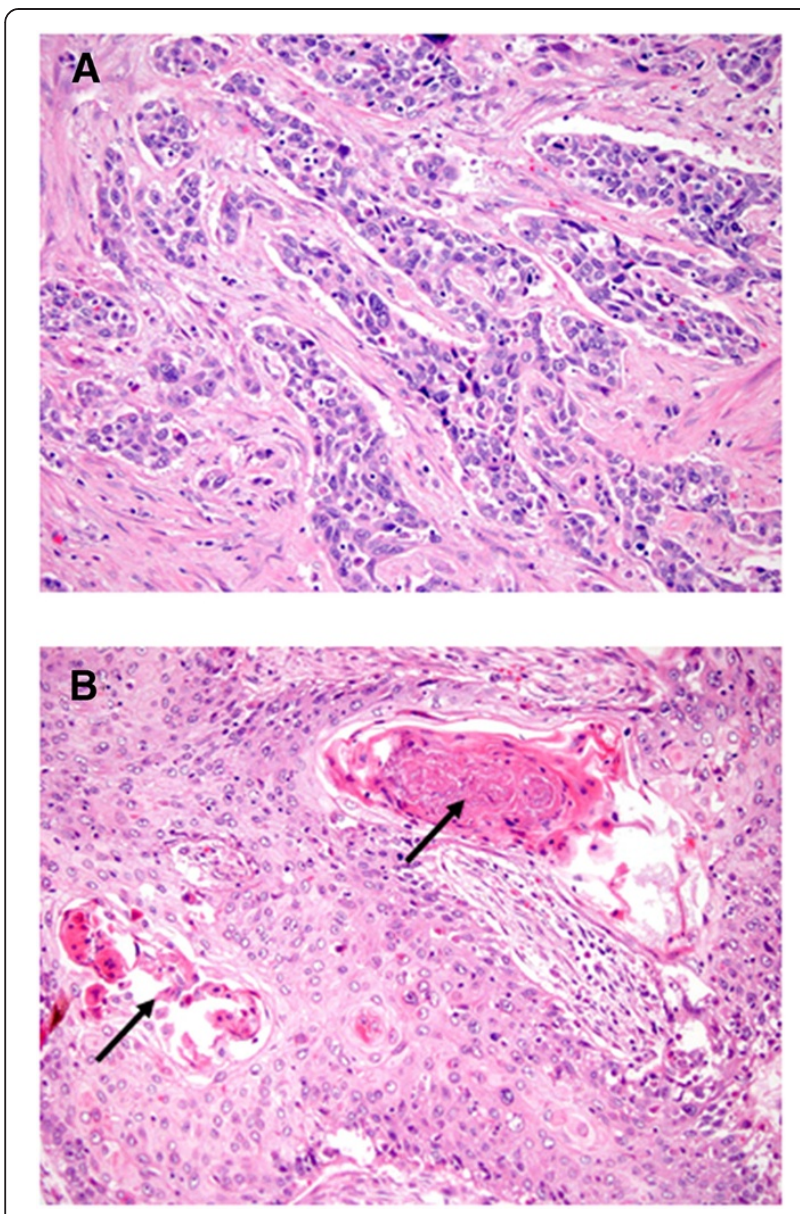

Figure 1 Two major subtypes of bladder cancer include (A) urothelial carcinoma, which is the most common form of bladder cancer and contains variably sized nests and (B) squamous cell carcinoma, which is characterized by desmosomes and keratin pearls (arrow).

forms of bladder cancer show differential clinical behavior, morphologic appearances, immunohistochemical markers and response to chemotherapy.

SCCa represents the second most common form of bladder cancer in the United States (5-8\% of bladder cancer cases) and is distinguished by invasive squamous carcinoma cells containing desmosomes and often keratin formation [3]. A higher incidence of bladder SCCa has been reported in parts of the Middle East and Africa, however, where chronic infection with the water-bourne parasite Schistosoma haematobium, represents a major risk factor in the development of this disease [4]. Other potential risk factors in the development of SCCa include longterm catheterization, calculi that form in the urinary tract, and a nonfunctioning bladder, amongst others, and these risk factors may account for many instances of SCCa identified in the United States and Europe.

Despite the distinct appearance of $\mathrm{UCa}$ and $\mathrm{SCCa}$, the cellular origin of these two forms of bladder cancer has been debated. Whereas UCa can arise in association with surface high-grade changes of the urothelium (e.g., carcinoma in situ) and SCCa can arise in association with squamous dysplasia and squamous carcinoma in situ of the bladder, there are numerous cases that show overlap of surface changes. For example, SCCa can occur in the absence of any surface squamous metaplasia and may only be associated with urothelial carcinoma in situ [3]. Furthermore, UCa itself has been shown to demonstrate "divergent" differentiation with the ability of UCa tumor cells to develop squamous or glandular features. These findings suggest that 1 ) the molecular relationship between historically distinct subtypes of bladder cancer may be more similar than previously hypothesized and 2) the surface urothelium in a bladder undergoing neoplastic alterations may be unusually suited to give rise to divergent phenotypes in the setting of both in situ and invasive disease.

To date, much of the molecular analysis on bladder SCCa has been limited due to a predominant focus on infectious, Schistosomal-derived cases as well as use of immortalized bladder cancer cell lines in a number of studies [5-8]. In this setting, the understanding of the relationship between $\mathrm{UCa}$ and $\mathrm{SCCa}$, as well as the distinction between primary molecular changes and those secondarily induced by infection-specific responses, becomes difficult. We sought to compare the two most common forms of pure bladder cancer in the US (UCa and non-infectious SCCa) using multi-level gene expression analysis to determine the relationship and possible hierarchy of these two examples of bladder cancer. The results from our study suggest a closer relationship between these neoplastic entities than previously proposed; a shared evolution of these cancers may represent an opportunity for targeting bladder cancer along common pathways early in the disease process.

\section{Methods}

\section{Specimen collection}

Specimens were collected with Institutional Review Board approval. The existing bladder cancer biobank (1971 onwards) was searched for snap-frozen tissue obtained from non-neoplastic bladder and/or ureter and from patients with either UCa or SCCa. Frozen sections were obtained from all specimens and reviewed; specimens with any necrosis or $<90 \%$ tumor or normal cell nuclei were excluded from analysis. H\&E slides corresponding to the initial pathology specimen associated with each sample were re-reviewed for accuracy of tumor classification. The clinical records for any patients with normal urothelium were reviewed; any patient with a precedent or subsequent occurrence of urinary tract neoplasia was excluded from analysis. This resulted in 8 normal urothelium specimens, $10 \mathrm{UCa}$ specimens and 9 
SCCa specimens (total in archive) used for analysis. No patient with SCCa had a precedent or concurrent history of Schistosomal infection. This study was approved by the Cleveland Clinic IRB.

\section{Raw gene expression levels}

Ten micrograms of total RNA from each sample was processed using the Affymetrix GeneChip one-cycle target labeling kit (Affymetrix, Santa Clara, CA). The resultant biotinylated cDNA was fragmented and subsequently hybridized to the GeneChip Human genome (54,675 probe sets including more than 35,000 human genes; Affymetrix). Arrays were washed, stained, and scanned using the Affymetrix Model 450 Fluidics Station and Affymetrix Model 3000 scanner per manufacturer's recommended protocols.

Expression values were generated using Microarray Suite (MAS) v5.0 software (Affymetrix). The probes were redefined according to a new study (http://brainarray. mhri.med.umich.edu/Brainarray/Database/CustomCDF/ version10) to combine probes representing the same gene for a single profile per gene. The hybridizations were normalized using the robust multichip averaging (rma) algorithm in the Bioconductor package affy (see http://www. bioconductor.org/) in order to obtain summary expression values for each probe set $[9,10]$. This resulted in more than 17,000 genes, each of which then has one numeric number to represent its relative gene expression intensity in the sample.

\section{Clustering study}

A hierarchical clustering algorithm was used to identify unsupervised clusters based on the Euclidean distance for dissimilarities between the data samples. The slightly modified "plot.phylo" program from analyses of phylogenetics and evolution (ape) package of $\mathrm{R}$ was used to show the clustering results $[11,12]$. The interquartile range (IQR) and coefficient of variation $(\mathrm{CV})$ were used to filter identified genes in the unsupervised clustering study. IQR was defined to be the distance between the third and first quartiles of the data; the $\mathrm{CV}$ of a vector was defined to be the standard deviation divided by its mean value. We used IQR $>0.3$ and $C V>0.05$ as our filtering criteria. This resulted in a data set of approximately 13600 genes. Other cutoff values provided similar clustering results. We also used the limma package to identify genes for supervised clustering analysis. When more than two classes of genes were present in the study group, the comparison was made between all pairs of classes. When comparison was made between two conditions, we used a fold change of 5 as a cutoff value to declare a gene significant.

We set 0.05 as our significance level for all tests. All calculations were implemented in $\mathrm{R}$ environment ( $\mathrm{R}>2.15 .0$, see http://www.r-project.org).

\section{Results}

\section{Comparative analysis}

Despite the shared urothelium from which SCCa and UCa arises, it is unclear whether these two morphologically distinct forms of bladder cancer share significant molecular overlap and, if so, whether a hierarchy in tumor types exists. In order to address this question, we performed a four-way interrogation of gene expression profiles: 1) normal urothelium versus SCCa, 2) normal urothelium versus $\mathrm{UCa}, 3)$ normal urothelium versus SCCa and UCa combined (shared alterations) and 4) UCa versus SCCa (divergent alterations). We included for analysis 8 samples of normal urothelium, 10 samples of invasive high-grade UCa and 9 samples of invasive SCCa. A boxplot of the data set shows that all samples have a roughly comparable distribution of the gene expression values, except only one sample (normal sample 1; Figure 2). When analyzed by subsequent unsupervised or supervised clustering studies, sample 1 did correctly segregate into the normal urothelial cluster; we therefore retained this sample in our study set.

Unexpectedly, the gene expression profiles revealed a large number of shared gene expression differences in

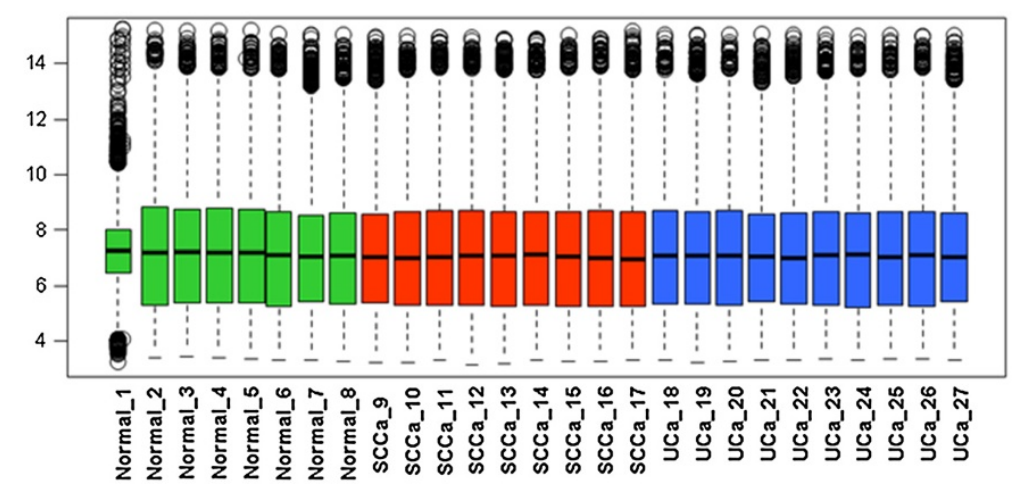

Figure 2 Boxplot of the 27 samples used for study presented in log2 scale. 


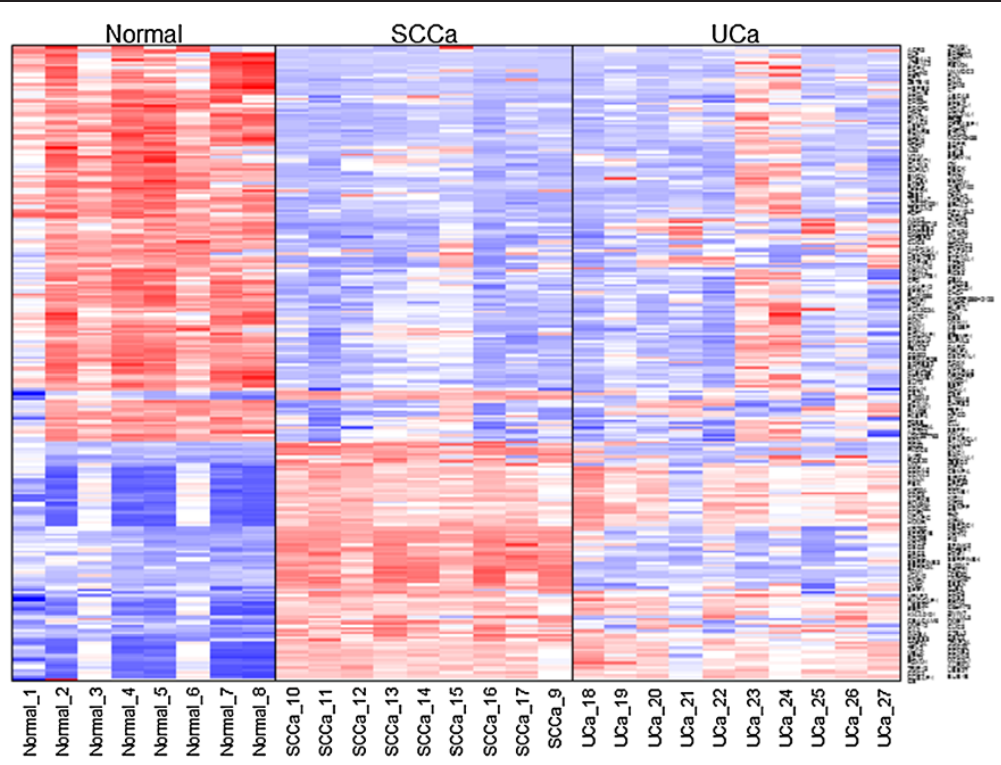

Figure 3 Heatmap comparing UCa and SCCa to normal urothelium for all 27 samples using supervised clustering and a fold change of 5, with 262 genes represented.

UCa and SCCa relative to the normal urothelium when using a 5-fold cut-off ( $\mathrm{n}=137$, Figure 3$)$. In addition to these shared gene expression differences, SCCa demonstrated an additional 366 uniquely dysregulated genes relative to normal urothelium, whereas UCa demonstrated only an additional 18 genes that were uniquely

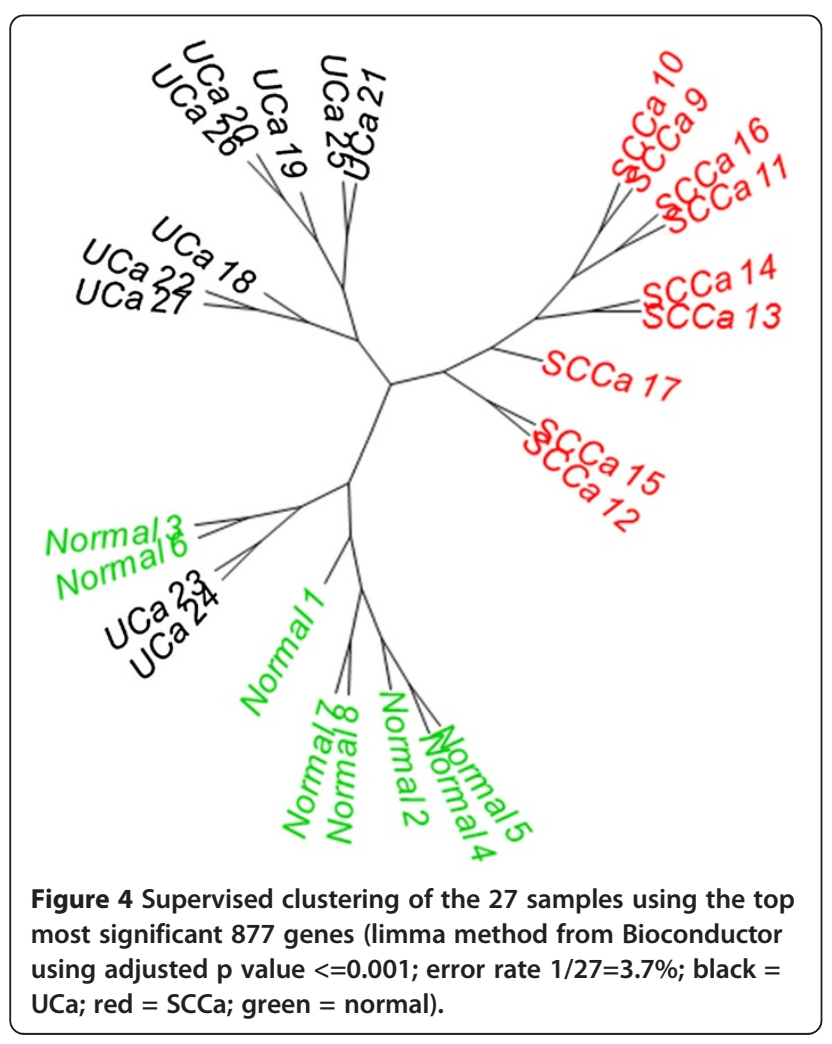

dysregulated relative to normal urothelium. Using supervised clustering (Figure 4) and unsupervised clustering (Figure 5A, B) analysis, we were able to reproducibly segregate normal urothelium, UCa and SCCa specimens, although two specimens (UCa23 and UCa24) appeared slightly different than other tumors in the UCa category, but could correctly segregate with other UCa specimens when a lower threshold value was applied to the analysis; specifically, no morphological difference was appreciated in these two specimens.

All differentially expressed genes were used to obtain fold changes (in $\log 2$ scale) to compare UCa versus normal and SCCa versus normal (Figure 6A). The majority of genes have fold change differences within $2(17,468$, $99.25 \%$, grey). A relatively larger number of genes have fold change differences above $2(184$, red) than the number of genes with fold change differences below -2 (47, blue). Overall, the fold change vectors correlated well with each other (cor. coefficient greater than 0.73), with the exception of the 184 genes located above the selected area (red), which are significantly higher in SCCa when compared to normal urothelium. A summary of the 4-way analysis performed with total gene expression differences is presented in Figure 6B.

\section{Commonly dysregulated genes in UCa and SCCa versus normal urothelium}

We next sought to determine commonalities in gene expression changes in $\mathrm{UCa}$ and SCCa versus normal urothelium. As normal urothelium lines the urinary tract throughout its length, and represents the common epithelium from which any form of bladder cancer (with 


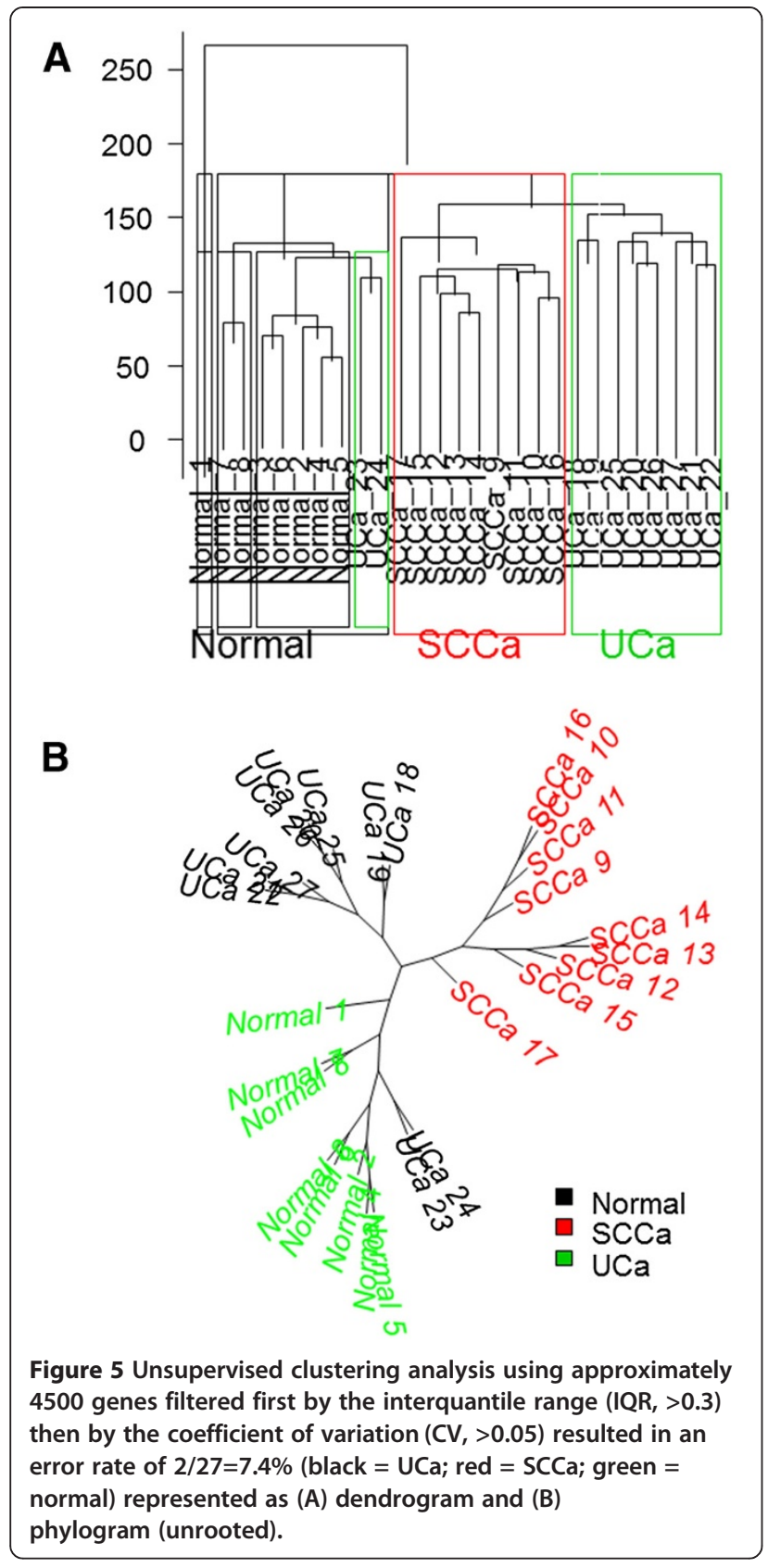

the exception of urachal carcinoma) derives, we queried whether shared pathways were commonly altered in these forms of bladder cancer. Using this rationale, we identified 137 genes that differed by at least 5 -fold in cancer specimens relative to normal urothelium (45 upregulated and 92 downregulated genes), with a representative subset containing functions related to cell growth and/or reported in cancer listed in Table 1.

The mitotic spindle checkpoint appeared generally upregulated, with overexpression of gene products of aurora kinase A (AURKA), aurora kinase B (AURKB), BUB1B, NUF2, MAD2L1, CCNB1, TPX2, ZWINT, ZWINT

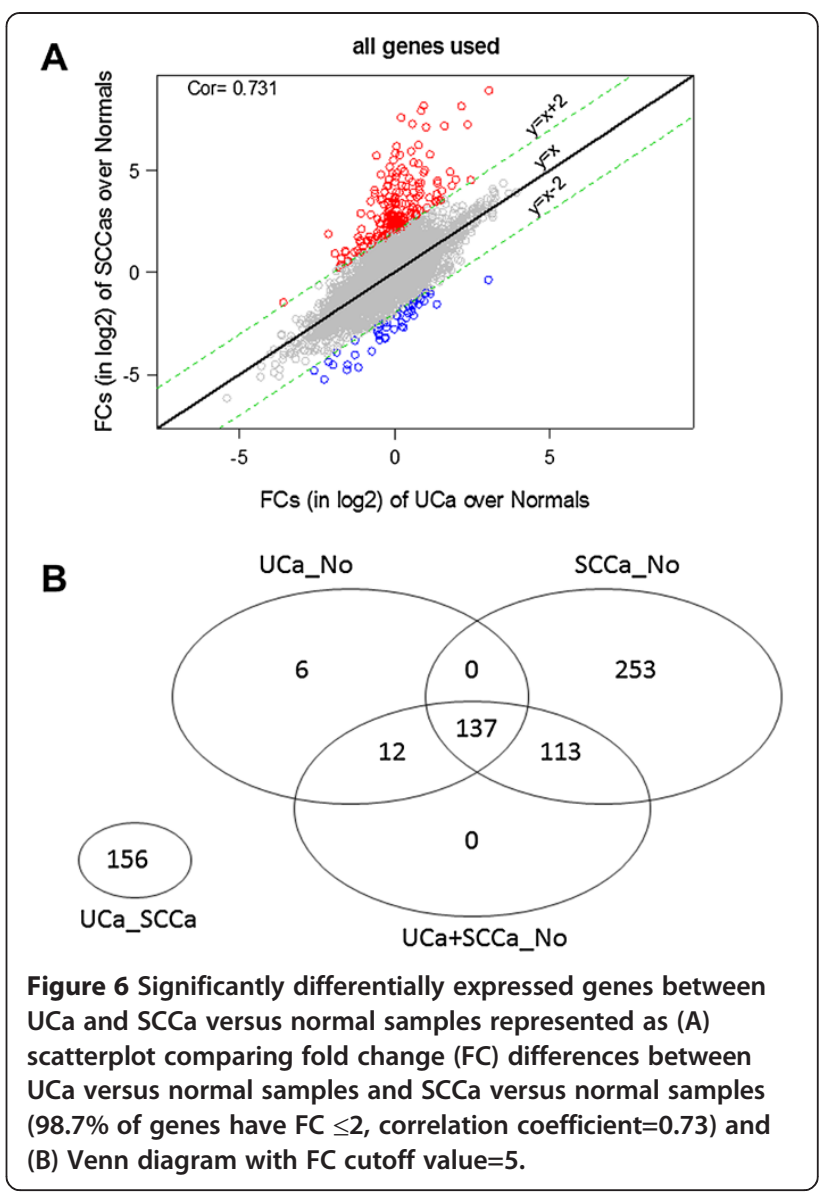

and $C D C 20$. Although these genes may be upregulated simply due to increased proliferative capacity of carcinomas, aurora kinase A has been previously investigated in UCa, where it is commonly found to be amplified [13] and may be a potential novel therapeutic target [14], which validates our results. A second category of upregulated genes included nicotine-responsive genes, as identified in both lung and head and neck squamous cancers, and include TTK, CEP55, PRC1 and FOXM1 [15-18]. As tobacco smoking is a preeminent risk factor for the development of both UCa and SCCa of the bladder, these genes may reflect this association. An additional category of overrepresented gene products included putative stem cell markers and/or mediators encoded by TTK, MELK, $D L G 7$, and $P B K$ [19-22]. Of note, very few pro-migratory factors were found to be upregulated in this shared population with the most likely pro-migration factor represented by RACGAP1, which encodes a small RhoGTPase.

Downregulated genes grouped into the major categories of inflammatory mediators [23], nicotine metabolizing genes, regulators or apoptosis and cell adhesion factors. Downregulated gene products include $C F D, C 7, D A R C$, PTX3, CD302, HLA-DQA1, CD69, P2RY14, SELE, JAM2, and CCL14, which include mediators of inflammatory cell 
Table 1 Commonly dysregulated genes shared by urothelial and squamous carcinoma versus normal rothelium

\begin{tabular}{|c|c|c|c|}
\hline Gene & Fold change UCa & Fold change SCCa & Function \\
\hline UBE2C & 15.1 & 15.1 & Ubiquitin-conjugating enzyme; promotes cell cycle; amplified in UCC \\
\hline TOP2A & 13 & 14.8 & DNA topoisomerase \\
\hline CDC20 & 11.4 & 21.1 & Regulates RUNX1; interacts with Aurora A; cell cycle regulation \\
\hline CCNB2 & 9.49 & 12.5 & Cyclin B2; TGF-beta mediated cell cyle control \\
\hline CEP55 & 9.32 & 15.1 & Centromeric protein regulated by FOXM1 \\
\hline TPX2 & 9.32 & 11.6 & Microtubule formation at kinetochores \\
\hline TTK & 9.08 & 10.7 & Mitotic spindle checkpoint protein; smoking responsive gene \\
\hline FOXM1 & 8.99 & 7.66 & Can increase levels of cyclin family members; smoking responsive gene \\
\hline ZWINT & 8.87 & 7.37 & Kinetochore interactor \\
\hline BUB1B & 8.83 & 9.06 & Kinase involved in spindle checkpoint function \\
\hline MELK & 8.58 & 12.4 & AMPK related kinase; implicated in stem cell function \\
\hline DLG7 & 7.98 & 12.3 & Putative stem cell factor that interacts with kinetochore; increased in UCC \\
\hline NUF2 & 7.64 & 7.97 & Kinetochore-associated protein involved in chromosome segregation \\
\hline MAD2L1 & 7.61 & 9.06 & Spindle checkpoint protein; regulates start of anaphase \\
\hline AURKB & 6.62 & 9.53 & Microtubule-associated protein involved in chromosome segregation \\
\hline CCNB1 & 6.43 & 9.89 & Cyclin B1; complexes with p34 to regulate mitotic activity \\
\hline RACGAP1 & 6.24 & 5.89 & RhoGTPase encoding gene \\
\hline AURKA & 5.64 & 8.51 & Kinetochore-associated protein found at spindle poles during mitosis \\
\hline $\mathrm{ADH} 1 \mathrm{~B}$ & -42.3 & -70.1 & Involved in tobacco smoke detoxification; implicated in esophageal cancer \\
\hline UPK1A & -14.4 & -22.4 & Urothelium-associated protein \\
\hline FHL1 & -12.5 & -8.37 & LIM-protein that regulates apoptosis and proliferation \\
\hline ANXA10 & -12.1 & -18.8 & Regulates cell growth; synergizes with p53 mutation for worse outcomes \\
\hline ADAMTS1 & -10.0 & -8.56 & Matrix metalloproteinase \\
\hline DARC & -9.93 & -15.9 & Binds cytokines; can influence tumor cell binding to endothelial cells \\
\hline MYH11 & -8.96 & -18.8 & Smooth muscle myosin \\
\hline DMN & -8.81 & -11.0 & Intermediate filament associated with desmin \\
\hline TCF21 & -7.43 & -9.92 & Tumor suppressor gene; undergoes methylation in some cancers \\
\hline HLA-DQA1 & -7.01 & -8.38 & Expression may be altered following repeated BCG exposure \\
\hline CD69 & -6.63 & -5.48 & Lectin superfamily; reduced in head and neck SCC patients \\
\hline ANK2 & -6.28 & -8.84 & Links integral membrane proteins to the underlying cytoskeleton \\
\hline CLU & -6.18 & -5.72 & Apoptotic mediators; expression decreased in many cancers \\
\hline SELE & -6.14 & -7.75 & Inflammatory mediator; regulates immune cell-endothelial interaction \\
\hline JAM2 & -6.07 & -7.61 & Tight junction protein; regulates immune cell binding \\
\hline $\mathrm{AOX} 1$ & -5.76 & -7.12 & Nicotine metabolizing protein \\
\hline PROM1 & -5.27 & -8.29 & Expressed in adult stem cells; mediates differentiation \\
\hline CCL14 & -5.09 & -8.40 & Cytokine \\
\hline
\end{tabular}

Differentially expressed genes have an adjusted $\mathrm{p}$ value of $<0.05$.

adhesion, humoral response and monocyte activity. HLA$D Q 1$ has been evaluated previously in UCa and its expression is associated with repeated exposure (and response) to BCG [24].

$A D H 1 B$ and $A O X 1$ are involved in the metabolism of nicotine, with the former gene implicated in the risk of esophageal carcinoma [25]. Apoptotic mediators that are downregulated include $C L U$, FHL1 and PCP4, whereas cell adhesion and cytoskeletal mediators that are downregulated include UPK1A, MYH11, DMN, MFAP4, ITM2A, ANK2, JAM2, MYLK, PROM1, DPT, and FBLN5.

Of the 137 genes differentially expressed between bladder UCa and SCCa versus normal urothelium, 18 have been previously reported to be up/down regulated in $\mathrm{UCa}$ and 35 have been reported in $\mathrm{SCCa}$ arising from non-bladder sites. Due to the rarity of profiling papers 
available on bladder SCCa, however, these factors have not been studied in this entity to date.

\section{A limited subset of uniquely dysregulated genes defines UCa}

One of the most surprising results from this study are the very small number of genes that were found to be uniquely dysregulated in UCa versus normal urothelium $(\mathrm{n}=18$; representative subset Table 2). The remainder of dysregulated genes $(n=137)$ are found in common with those altered in bladder SCCa. Uniquely dysregulated genes in UCa include CLCA4 (chloride channel), IL33, GPR171 (G protein coupled receptor), CENPF (centromere protein F) and $C D 36$ (thrombospondin receptor). $E Z H 2$ has been reported to be upregulated in UCa and represents a putative stem cell marker [26] and a repressor of E-cadherin expression [27]; of relevance, E-cadherin is frequently lost in high-grade UCa [28].

\section{Well-categorized squamous factors are uniquely upregulated in SCCa}

Finally, we analyzed uniquely dysregulated genes in SCCa versus normal urothelium and identified 185 upregulated and 181 downregulated unique genes that differed by at least 5 -fold between these two groups (representative subset presented in Table 3). The majority of dysregulated genes are factors that have been associated with the squamous phenotype and histology, with many of these factors identified in squamous carcinomas arising at other sites. Upregulated gene products include keratins that are specific for squamous epithelium (KRT6B, KRT16, KRT5, $K R T 20$ ), the family of $\mathrm{S} 100$ calcium binding proteins commonly upregulated in SCCa from various anatomical sites (S100A7, S100A8, S100A9, S100A12, S100A14, S100A16), the serpin family (SERPINB1-7), desmosome-associated proteins that characterize squamous epithelium (DSG1, DSG3, PKP1, PKP3), numerous peptides (PI3, SPINK5, $K L K 7, S L P I)$, and a variety of pro-motility factors. Downregulated gene products include putative tumor suppressor genes (SCUBE2), factors previously reported as lost in aggressive bladder cancer (FOXA1, GATA3, UPK $3 A$ ), and metabolizing enzymes with polymorphisms affecting cancer risk (UGT1A10, UGT1A7).

\section{Discussion}

Current pathological classification distinguishes UCa and $\mathrm{SCCa}$ as distinct diagnostic entities [4]. This has resulted in numerous publications that have evaluated the differences in clinical outcomes, treatment response and molecular profiles that distinguish these two bladder cancer types, with mixed results [29]. Although some studies have suggested that when compared stage-for-stage, the outcomes are similar for patients with bladder UCa and SCCa $[3,29,30]$, other studies have implicated that SCCa and/or UCa with squamous differentiation may present at a higher stage and behave more aggressively [31-33]. One consistency amongst studies is the limited response of bladder SCCa of the bladder to conventional chemotherapy and/or radiation therapy that is administered in the setting of UCa and may relate to the squamous phenotype

Table 2 Uniquely dysregulated genes in grothelial garcinoma versus normal urothelium

\begin{tabular}{lcl}
\hline Gene & Fold change UCa & Function \\
\hline MEST & 8.14 & Encodes a member of alpha/beta hydrolase superfamily; imprinting in cancer \\
EZH2 & 5.57 & Stem cell related gene; previously reported as upregulated in UCa \\
CENPF & 5.54 & Component of centromere/kinetochore complex; affects chromosome segregation \\
GINS2 & 5.43 & Psf2 homolog; complex component that regulates DNA replication in yeast \\
CLCA4 & -11.9 & Member of the calcium sensitive chloride conductance superfamily \\
POU2AF1 & -8.96 & Regulates TH1 and TH2 immune responses \\
IL33 & -7.99 & Member of IL1 family; enhances TH2 cytokine production \\
SEPP1 & -6.94 & Selenoprotein; may affect oxidative stress response \\
IL6 & -6.57 & Inflammatory cytokine; increased after BCG administration in the bladder \\
GPR171 & -6.45 & G protein-coupled receptor; may regulate hematopoietic progenitor cells \\
FOSB & -6.26 & One member of the AP-1 transcription factor complex; implicated in ovarian carcinoma \\
ITK & -6.05 & Regulator of T cell proliferation \\
AGR3 & -5.62 & May regulate protein folding; may regulate cisplatin resistance in ovarian cancer \\
CCL19 & -5.45 & Cytokine affecting B and T cell migration; may enhance B-cell mediated immunity; \\
CD36 & -5.42 & Thrombospondin receptor; may affect tumor vascularity and matrix content \\
DKK1 & -5.33 & Inhibits WNT signaling; may inhibit invasive behavior and self-renewal in some cancers \\
\hline DEfent
\end{tabular}


Table 3 Uniquely dysregulated genes in squamous cell carcinoma versus normal urothelium

\begin{tabular}{|c|c|c|}
\hline Gene & Fold change SCCa & Function \\
\hline KRT6B & 289 & High molecular weight cytokeratin; stratified epithelium differentiation \\
\hline S100A7 & 242 & Promotes migration and invasion of SCCa; reported in urine of bladder SCCa patients \\
\hline KRT16 & 147 & High molecular weight cytokeratin \\
\hline $\mathrm{Pl} 3$ & 140 & Peptidase inhibitor 3/elafin; marker of abnormal squamous growth; induced by inflammation \\
\hline DSG3 & 77.6 & $\begin{array}{l}\text { Desmosome-associated protein; upregulated in } \\
\text { various forms of SCCa }\end{array}$ \\
\hline SERPINB4 & 58.3 & Member of human SCCa antigen locus; activated by STAT3; enhances survival of SCCa cells \\
\hline CNFN & 56.3 & Cornifelin; squamous epithelial marker \\
\hline KLK10 & 53.9 & Serine protease; increased in oral and lung SCCa \\
\hline SERPINB3 & 42.5 & Member of human SCCa antigen locus; activated by STAT3; enhances survival of SCCa cells \\
\hline FGFBP1 & 31.3 & FGF carrier protein; promotes angiogenesis in SCCa from various sites \\
\hline MMP1 & 31.0 & Matrix metalloproteinase that degrades collagens; induced by EGF in bladder cancer patients \\
\hline DSG1 & 29.6 & Desmosome-associated protein; enhances loss of cell adhesion in SCCa from various sites \\
\hline KRT5 & 25.8 & High-molecular weight cytokeratin; expressed by squamous epithelium \\
\hline $\mathrm{DSC3}$ & 25.1 & $\begin{array}{l}\text { Desmosome-associated protein; marker of squamous differentiation; can } \\
\text { inhibit EGFR pathway }\end{array}$ \\
\hline S100A8 & 22.9 & May predict metastatic potential of bladder cancer; increased in SCCa from various sites \\
\hline LY6D & 21.1 & Affects interaction between SCCa cells and endothelial cells \\
\hline CA2 & 19.3 & Carbonic anhydrase II; Previously reported in bladder SCCa \\
\hline MMP10 & 18.7 & Matrix metalloproteinase; activated by EGF and STAT3; inhances SCCa invasion at other sites \\
\hline PTHLH & 15.5 & Parathyroid hormone-like hormone; previously reported in bladder SCCa; may affect apoptosis \\
\hline SCEL & 15.1 & Squamous epithelium marker \\
\hline CALB1 & 12.9 & Calbindin 1 ; calcium binding protein of the troponin $C$ superfamily \\
\hline PTPRZ1 & 11.9 & Receptor protein tyrosine phosphatase; involved in CNS development \\
\hline LAMC2 & 8.95 & Laminin, gamma2; Overexpressed in esophageal SCC \\
\hline KIF14 & 6.58 & Microtubule motor protein; reported in laryngeal carcinoma \\
\hline JUP & 6.31 & Associated with both desmosomes and intermediate junctions \\
\hline SCUBE2 & -27.1 & Secreted protein; putative tumor suppressor in breast cancer \\
\hline HPGD & -20.6 & Metabolism of prostaglandins; downregulated in gastric cancer by COX2 \\
\hline HMGCS2 & -15.6 & HMG-CoA synthase family; enzyme involved in ketogenesis \\
\hline CYP4B1 & -15.0 & Involved in drug metabolism and lipid synthesis; related to bladder cancer risk in one study \\
\hline TGFBR3 & -13.1 & Encodes TGF- $\beta$ receptor III; reduced expression in numerous cancers \\
\hline UPK3A & -10.9 & Uroplakin 3A; urothelial marker; loss of expression associated with aggressive bladder cancer \\
\hline $\mathrm{AOC} 3$ & -10.1 & Copper amine oxidase; aids in leukocyte adhesion and transmigration \\
\hline FBLN1 & -9.86 & Secreted glycoprotein; may be involved in ECM remodeling; downregulated in gastric cancer \\
\hline FOXA1 & -9.82 & Forkhead class of DNA binding proteins; loss occurs in aggressive bladder cancer \\
\hline PPARG & -9.27 & $\begin{array}{l}\text { Regulate gene transcription together with retinoid } X \text { receptors; may } \\
\text { influence } B C G \text { response }\end{array}$ \\
\hline GATA3 & -8.97 & Transcription factor; downregulated in bladder cancer \\
\hline CCL15 & -8.63 & Chemotactic for T cells and monocytes; \\
\hline TSPAN8 & -7.96 & Tetraspanin family; interacts with integrins; may regulate motility in various cancers \\
\hline ADRA2A & -7.75 & Alpha-2-adrenergic receptor; \\
\hline PDGFD & -7.74 & $\begin{array}{l}\text { PDGF family; can regulate motility in many cancer types and } \\
\text { chemotaxis }\end{array}$ \\
\hline KRT20 & -6.32 & Low molecular weight cytokeratin; typically patchy expression in urothelial carcinoma \\
\hline
\end{tabular}


Table 3 Uniquely dysregulated genes in squamous cell carcinoma versus normal urothelium (Continued)

\begin{tabular}{lll}
\hline CYP1A1 & -5.7 & $\begin{array}{l}\text { Hypermethylated in bladder cancer; polymorphisms related to bladder } \\
\text { cancer risk }\end{array}$ \\
CYP1B1 & -5.61 & Polymorphisms associated with bladder cancer risk \\
UGT1A10 & -5.28 & UDP-glucuronosyltransferase; involved in detoxification of carcinogens \\
UGT1A7 & -5.28 & UDP-glucuronosyltransferase; polymorphisms associated with cancer risk \\
\hline
\end{tabular}

Differentially expressed genes have an adjusted $p$ value of $<0.05$.

$[34,35]$. To date, however, the relationship between these two forms of bladder cancer that arise from the urothelial lining of the bladder has not been clearly delineated.

The results from our study suggest that UCa generally shares the majority of its dysregulated genes relative to normal urothelium in common with SCCa, with very few uniquely dysregulated genes; in contrast, SCCa while sharing many genes in common with $\mathrm{UCa}$ - shows a much larger category of dysregulated genes that are often in common with SCCa arising at other sites. When considering the relationship between these two closely related entities, two possibilities emerge. First, invasive UCa may represent a default pathway of bladder cancer development, with clonal change resulting in SCCa development and overgrowth of a pre-existent UCa. This hypothesis is supported by the not infrequent finding of mixed morphology bladder cancers, where a welldocumented UCa contains areas of squamous and/or glandular differentiation [4]. Further supporting this hypothesis is a prior paper that has examined the relationship of co-existent small cell carcinoma and UCa of the bladder: the results from this prior study suggest that the small cell carcinoma in this setting represented a clonal outgrowth from the background invasive $\mathrm{UCa}$ [36] a finding that might not be dissimilar across all other bladder cancer "subtypes" and which can be supported by the findings in this paper. A second possibility is that an early bladder cancer stem cell exists, either prior to invasion or early in the course of invasion, which gives rise to distinct morphological entities along discrete molecular lineages that are considered pure subtypes. Specifically, early molecular changes define a number of shared alterations between various bladder cancer subtypes that subsequently diverge along different morphologic lines [36]. In such a scenario, the limited number of additional alterations identified in $\mathrm{UCa}$ would suggest this to be a "default" pathway in bladder carcinogenesis, with significant additional alterations required to develop the squamous phenotype.

Regardless of the model proposed, the current data supports a close evolution between UCa and SCCa, with gene expression changes in the latter primarily reflecting morphological correlates of the squamous phenotype seen in $\mathrm{SCCa}$ arising from different sites. Our data also suggest that proliferative changes, including deregulation (and in some cases amplification) of mitotic spindle checkpoint components may be critical in the early stages of bladder tumorigenesis. Further validation of our findings using other "pure" types of bladder cancer - such as adenocarcinoma and small cell carcinoma - will further strengthen the implications of our results, although the rare nature of these other forms of bladder cancer may make such a study challenging.

Although we have used only one technique to analyze the relationship between UCa and SCCa, our ability to reproducibly segregate the entities in our study using both supervised and unsupervised clustering analysis suggest that our data is robust. A second limitation is the use of a limited number of specimens for analysis, although the use of $10 \mathrm{SCCa}$ samples is relatively high given the rarity of this disease entity. The overall gene expression profiles between our two bladder cancer entities suggest that the development of these bladder cancer forms occurs along similar lines. However, it is clear that the magnitude of expression changes differs in some instances; for example, reduction in $A D H 1 B$ occurs by a factor of 40-fold in UCa and 70-fold in SCCa. The importance of relative fold change (versus directionality) in these cancer subtypes was not a primary focus of investigation in this study. However, the relative increase or decrease in mRNA expression may have a relevant biological role when studied in a whole cell system.

Our study has identified numerous categories of genes that may be of relevance to the development of $\mathrm{UCa}$, including mitotic spindle regulators, putative stem cell factors, nicotine metabolizing enzymes and inflammatory regulators. The vast majority of these genes appear to be similarly dysregulated in bladder SCCa. One exception is a large category of inflammatory mediators (POU2AF1, IL33, IL6, ITK, CCL19) that are altered and may reflect the administration of BCG, which is frequently given intravesically for superficial UCa but not SCCa. In contrast, SCCa appears to overlap significantly in gene expression differences with UCa and additionally contains a large number of additional up- and downregulated gene products. Perhaps not surprisingly, many of these gene transcripts have been reported in SCCa from the head and neck region, oral cavity, lung and skin. As SCCa is considered to have limited response to 
therapies conventionally employed for $\mathrm{UCa}$, the broad list of discrete targets - some of which are currently undergoing clinical trials for targeted therapy in other forms of SCCa - may provide an alternative treatment for patients with either pure or mixed SCCa of the bladder.

\section{Conclusions}

In summary, we found that UCa and SCCa of the bladder share a number of differentially regulated genes, suggesting a close evolution of these two major subtypes of bladder cancer. Future studies that seek to further delineate the relationship and, thus, pathogenesis of various forms of bladder cancer will provide additional insight into the development of bladder cancer. Ultimately, the finding of shared molecular changes may allow investigators to develop targeted therapy that may be used either earlier in the course of disease or treat a broader range of cancer morphologies with success.

\section{Competing interests}

The authors declare that they have no competing interests.

\section{Authors' contributions}

DEH collected the specimens, performed pathologic analysis, interpreted the data and drafted the manuscript; ZZ performed data analysis and drafted the manuscript; DP performed gene expression analysis and reviewed the manuscript; BTT analyzed the data and reviewed the manuscript. All authors read and approve of the final manuscript.

\section{Acknowledgments}

We thank the Cleveland Clinic for providing bladder samples for analysis. We thank Jill Barnholtz-Sloan, PhD, for careful review and input to this manuscript.

\section{Author details}

'Department of Pathology, University of California at San Diego, 9500 Gilman Drive, MC 0612, La Jolla, CA 92093, USA. Van Andel Research Institute, Grand Rapids, MI, USA. ${ }^{3}$ National Cancer Centre of Singapore-VARI, Singapore, Singapore. ${ }^{4}$ Duke-NUS Graduate Medical School, Singapore, Singapore.

Received: 6 June 2013 Accepted: 11 October 2013

Published: 17 October 2013

\section{References}

1. Wu XR: Urothelial tumorigenesis: a tale of divergent pathways. Nat Rev Cancer 2005, 5:713-725.

2. Amin MB, McKenney JK, Paner GP, Hansel DE, Grignon DJ, Montironi R, Lin $\mathrm{O}$, Jorda M, Jenkins LC, Soloway M, et al: ICUD-EAU international consultation on bladder cancer 2012: pathology. Eur Urol 2013, 63:16-35.

3. Lagwinski N, Thomas A, Stephenson AJ, Campbell S, Hoschar AP, El-Gabry E, Dreicer R, Hansel DE: Squamous cell carcinoma of the bladder: a clinicopathologic analysis of 45 cases. Am J Surg Pathol 2007, 31:1777-1787.

4. Eble JN, Sauter G, Epstein II, Sesterhenn IA: Pathology and genetics of tumours of the urinary system and male genital organs. Lyon, France: IARC Press; 2004

5. Ewis AA, El-Samman E, Ali N, Kajimoto K, Shinohara Y, Ishikawa M, Kanayama HO, Baba Y: Gene expression profile in squamous cell carcinoma of the urinary bladder using complementary deoxyribonucleic acid microarray. Urol Oncol 2007, 25:120-127.

6. Blaveri E, Simko JP, Korkola JE, Brewer JL, Baehner F, Mehta K, Devries S, Koppie T, Pejavar S, Carroll P, et al: Bladder cancer outcome and subtype classification by gene expression. Clin Cancer Res 2005, 11:4044-4055.

7. Sanchez-Carbayo M, Socci ND, Charytonowicz E, Lu M, Prystowsky M, Childs G, Cordon-Cardo C: Molecular profiling of bladder cancer using CDNA microarrays: defining histogenesis and biological phenotypes. Cancer Res 2002, 62:6973-6980.

8. Ostergaard M, Rasmussen HH, Nielsen HV, Vorum H, Orntoft TF, Wolf H, Celis JE: Proteome profiling of bladder squamous cell carcinomas: identification of markers that define their degree of differentiation. Cancer Res 1997, 57:4111-4117.

9. Zhang Z, Furge KA, Yang XJ, Teh BT, Hansel DE: Comparative gene expression profiling analysis of urothelial carcinoma of the renal pelvis and bladder. BMC Med Genomics 2010, 3:58.

10. Bolstad BM, Irizarry RA, Astrand M, Speed TP: A comparison of normalization methods for high density oligonucleotide array data based on variance and bias. Bioinformatics 2003, 19:185-193.

11. Irizarry RA, Bolstad BM, Collin F, Cope LM, Hobbs B, Speed TP: Summaries of affymetrix GeneChip probe level data. Nucleic Acids Res 2003, 31:e15.

12. Paradis E, Claude J, Strimmer K: APE: analyses of Phylogenetics and Evolution in R language. Bioinformatics 2004, 20:289-290.

13. Sen $S$, Zhou H, Zhang RD, Yoon DS, Vakar-Lopez F, Ito S, Jiang F, Johnston D, Grossman HB, Ruifrok AC, et al: Amplification/overexpression of a mitotic kinase gene in human bladder cancer. J Natl Cancer Inst 2002, 94:1320-1329.

14. Zhou N, Singh K, Mir MC, Parker Y, Lindner D, Dreicer R, Ecsedy JA, Zhang Z, Teh BT, Almasan A, et al: The investigational aurora kinase a inhibitor MLN8237 induces defects in cell viability and cell-cycle progression in malignant bladder cancer cells in vitro and in vivo. Clin Cancer Res 2013, 19:1717-1728.

15. Landi MT, Dracheva T, Rotunno M, Figueroa JD, Liu H, Dasgupta A, Mann FE, Fukuoka J, Hames M, Bergen AW, et al: Gene expression signature of cigarette smoking and its role in lung adenocarcinoma development and survival. PLOS One 2008, 3:e1651.

16. Kim IM, Ackerson T, Ramakrishna S, Tretiakova M, Wang IC, Kalin TV, Major ML, Gusarova GA, Yoder HM, Costa RH, et al: The forkhead Box m1 transcription factor stimulates the proliferation of tumor cells during development of lung cancer. Cancer Res 2006, 66:2153-2161.

17. Gemenetzidis E, Bose A, Riaz AM, Chaplin T, Young BD, Ali M, Sugden D, Thurlow JK, Cheong SC, Teo SH, et al: FOXM1 upregulation is an early event in human squamous cell carcinoma and it is enhanced by nicotine during malignant transformation. PLoS One 2009, 4:e4849.

18. Waseem A, Ali M, Odell EW, Fortune F, Teh MT: Downstream targets of FOXM1: CEP55 and HELLS are cancer progression markers of head and neck squamous cell carcinoma. Oral Oncol 2010, 46:536-542.

19. Nakano I, Paucar AA, Bajpai R, Dougherty JD, Zewail A, Kelly TK, Kim KJ, Ou J, Groszer M, Imura T, et al: Maternal embryonic leucine zipper kinase (MELK) regulates multipotent neural progenitor proliferation. J Cell Biol 2005, 170:413-427.

20. Gudmundsson KO, Thorsteinsson L, Sigurjonsson OE, Keller JR, Olafsson K, Egeland T, Gudmundsson S, Rafson T: Gene expression analysis of hematopoietic progenitor cells identifies Dlg7 as a potential stem cell gene. Stem Cells 2007, 25:1498-1506.

21. Dougherty JD, Garcia AD, Nakano I, Livingstone M, Norris B, Polakiewicz R, Wexler EM, Sofroniew MW, Kornblum HL, Geschwind DH: PBK/TOPK, a proliferating neural progenitor-specific mitogen-activated protein kinase kinase. J Neurosci 2005, 25:10773-10785.

22. Badenhorst P: Tramtrack controls glial number and identity in the drosophila embryonic CNS. Development 2001, 128:4093-4101.

23. Yang H, Gu J, Lin X, Grossman HB, Ye Y, Dinney CP, Wu X: Profiling of genetic variations in inflammation pathway genes in relation to bladder cancer predisposition. Clin Cancer Res 2008, 14:2236-2244.

24. Saban MR, Hellmich HL, Simpson C, Davis CA, Lang ML, Ihnat MA, O'Donnell MA, Wu XR, Saban R: Repeated BCG treatment of mouse bladder selectively stimulates small GTPases and HLA antigens and inhibits single-spanning uroplakins. BMC Cancer 2007, 7:204.

25. Tanaka F, Yamamoto K, Suzuki S, Inoue H, Tsurumaru M, Kajiyama Y, Kato $H_{\text {, }}$ Igaki H, Furuta K, Fujita $H$, et al: Strong interaction between the effects of alcohol consumption and smoking on oesophageal squamous cell carcinoma among individuals with $\mathrm{ADH} 1 \mathrm{~B}$ and/or $\mathrm{ALDH} 2$ risk alleles. Gut 2010, 59:1457-1464.

26. Wang H, Albadine R, Magheli A, Guzzo TJ, Ball MW, Hinz S, Schoenberg MP, Netto GJ, Gonzalgo ML: Increased EZH2 protein expression is associated with invasive urothelial carcinoma of the bladder. Urol Oncol 2012, 30:428-433.

27. Cao Q, Yu J, Dhanasekaran SM, Kim JH, Mani RS, Tomlins SA, Mehra R, Laxman B, Cao X, Yu J: Repression of E-cadherin by the polycomb group protein EZH2 in cancer. Oncogene 2008, 27:7274-7284. 
28. Bryan RT, Tselepis C: Cadherin switching and bladder cancer. J Urol 2010, 184:423-431.

29. Rogers CG, Palapattu GS, Shariat SF, Karakiewicz PI, Bastian PJ, Lotan Y, Gupta A, Vazina A, Gilad A, Sagalowsky Al, et al: Clinical outcomes following radical cystectomy for primary nontransitional cell carcinoma of the bladder compared to transitional cell carcinoma of the bladder. J Urol 2006, 175:2048-2053. discussion 2053.

30. Nishiyama H, Habuchi T, Watanabe J, Teramukai S, Tada H, Ono Y, Ohshima S, Fujimoto K, Hirao Y, Fukushima M, et al: Clinical outcome of a large-scale multi-institutional retrospective study for locally advanced bladder cancer: a survey including 1131 patients treated during 1990-2000 in Japan. Eur Urol 2004, 45:176-181.

31. Debbagh A, Bennani S, Hafiani M, el Mrini M, Benjelloun S: Epidermoid carcinoma of the bladder. Apropos of 14 cases. Ann Urol (Paris) 1997, 31:199-203.

32. Johnson DE, Schoenwald MB, Ayala AG, Miller LS: Squamous cell carcinoma of the bladder. J Urol 1976, 115:542-544

33. Scosyrev E, Yao J, Messing E: Urothelial carcinoma versus squamous cell carcinoma of bladder: is survival different with stage adjustment? Urology 2009, 73:822-827.

34. Rundle JS, Hart AJ, McGeorge A, Smith JS, Malcolm AJ, Smith PM: Squamous cell carcinoma of bladder. A review of 114 patients. Br J Urol 1982, 54:522-526.

35. Khaled HM, Hamza MR, Mansour O, Gaafar R, Zaghloul MS: A phase II study of gemcitabine plus cisplatin chemotherapy in advanced bilharzial bladder carcinoma. Eur J Cancer 2000, 36(Suppl 2):34-37.

36. Cheng L, Jones TD, McCarthy RP, Eble JN, Wang M, MacLennan GT, Lopez-Beltran A, Yang XJ, Koch MO, Zhang S, et al: Molecular genetic evidence for a common clonal origin of urinary bladder small cell carcinoma and coexisting urothelial carcinoma. Am J Pathol 2005, 166:1533-1539.

doi:10.1186/1755-8794-6-42

Cite this article as: Hansel et al:: Gene profiling suggests a common evolution of bladder cancer subtypes. BMC Medical Genomics 2013 6:42.

\section{Submit your next manuscript to BioMed Central and take full advantage of:}

- Convenient online submission

- Thorough peer review

- No space constraints or color figure charges

- Immediate publication on acceptance

- Inclusion in PubMed, CAS, Scopus and Google Scholar

- Research which is freely available for redistribution

Submit your manuscript at www.biomedcentral.com/submit
(O) Biomed Central 